\title{
A percepção do usuário em relação à qualidade do atendimento na atenção primária à saúde
}

\author{
The user's perception in relation to the quality of primary health care attendance \\ La percepción del usuario em la relación com la calidad de servicio em la atención \\ primaria de salud
}

Isabella Cristina Oliveira Morais ${ }^{1}$, Leticia Aparecida Resende Padilha1 ${ }^{1}$ Nathália Resende Pereira Vaz $^{1}$, Renata Licursi Nogueira ${ }^{1 *}$.

\section{RESUMO}

Objetivo: Avaliar os atributos referenciados pelos princípios e diretrizes do Sistema Único de Saúde (SUS) sob a perspectiva dos usuários para mensurar a qualidade dos serviços oferecidos, evidenciando assim, o nível de conhecimento sobre o assunto. Métodos: Estudo analítico, transversal, cujos dados foram colhidos pela aplicação do questionário PCATool - Brasil, em usuários aleatórios nas salas de espera de cinco Unidades Básicas de Saúde da cidade de Franca-SP, totalizando 100 entrevistados. Resultados: Nas unidades avaliadas, o número de mulheres entrevistadas foi maior do que o de homens e a idade predominante variou em torno de 38 anos. Em relação aos atributos, não foram encontradas diferenças significativas entre os escores. O pior escore encontrado foi a Integralidade - dimensão serviços prestados-e o único atributo orientado para Atenção Primária à Saúde (APS) foi o acesso de primeiro contato. Conclusão: Há necessidade de melhorar o acesso da população aos serviços de saúde e reforçar a importância da educação e conscientização sobre o funcionamento da APS, para que os usuários tenham melhor entendimento desta como porta de entrada do SUS, fortalecendo, assim, os objetivos fundamentais deste sistema: promover saúde e prevenir doenças.

Palavras-chave: Atenção primária, Serviços de saúde, Sistema único de saúde.

\begin{abstract}
Objective: To evaluate the attributes referenced by the principles and guidelines of the Unified Health System (SUS) from the perspective of users to measure the quality of the services offered, thus evidencing the level of knowledge on the subject. Methods: Cross-sectional analytical study, whose data were collected by applying the PCATool - Brazil questionnaire, in random users in the waiting rooms of five Basic Health Units in the city of Franca-SP, totaling 100 respondents. Results: In the evaluated units, the number of women interviewed was greater than that of men and the predominant age varied around 38 years. Regarding the attributes, no significant differences were found between the scores. The worst score found was Comprehensiveness - dimension of services provided - and the only attribute oriented to Primary Health Care (PHC) was first contact access. Conclusion: There is a need to improve the population's access to health services and reinforce the importance of education and awareness about the functioning of PHC, so that users have a better understanding of it as a gateway to SUS, thus strengthening the fundamental objectives of this system: promoting health and preventing disease.
\end{abstract}

Key words: Primary care, Health services, Unified health system

\footnotetext{
1 Universidade de Franca (UNIFRAN), Franca - São Paulo.

*E-mail: renolic@gmail.com
} 


\section{RESUMEN}

Objetivo: Evaluar los atributos referenciados por los principios y lineamientos del Sistema Único de Salud (SUS) desde la perspectiva de los usuarios para medir la calidad de los servicios ofrecidos, evidenciando así el nivel de conocimiento sobre el tema. Métodos: Estudio analítico transversal, cuyos datos fueron recolectados aplicando el cuestionario PCATool - Brasil, en usuarios aleatorios en las salas de espera de cinco Unidades Básicas de Salud en la ciudad de Franca-SP, con un total de 100 encuestados. Resultados: En las unidades evaluadas, el número de mujeres entrevistadas fue mayor que el de los hombres y la edad predominante varió alrededor de los 38 años. En cuanto a los atributos, no se encontraron diferencias significativas entre los puntajes. El peor puntaje encontrado fue la integralidad (dimensión de los servicios prestados) y el único atributo orientado a la Atención Primaria de Salud (APS) fue el acceso por primer contacto. Conclusión: Es necesario mejorar el acceso de la población a los servicios de salud y reforzar la importancia de la educación y la conciencia sobre el funcionamiento de la APS, para que los usuarios lo entiendan mejor como una puerta de entrada al SUS, fortaleciendo así los objetivos fundamentales de este sistema: promoviendo la salud y previniendo enfermedades.

Palabras clave: Atención primária, Servicios de salud, Sistema de salud unificado.

\section{INTRODUÇÃO}

Previamente à criação do Sistema Único de Saúde (SUS), a sistematização dos serviços de saúde no Brasil era extremamente complicada, já que não existia uma coordenação entre os diversos sistemas que compunham a organização sanitária. Com isso, os recursos eram disperdiçados, resultando em dificuldades para resolver os problemas da população. Havia uma dicotomia entre as ações de saúde e as de assistência médico-hospitalar. Até a década de 1920, a saúde pública era uma espécie de assistencialismo exclusivo ou modelo residual (PAIM JS, 2009).

Embora o direito ao bem-estar tenha sido difundido internacionalmente desde a criação da Organização Mundial da Saúde (OMS), em 1948, somente quarenta anos depois, através da Constituição Brasileira de 1988, o Brasil reconheceu formalmente a saúde como direito social (ESCOLA NACIONAL DE SAÚDE PÚBLICA SÉRGIO AROUCA, 2009).

Nesse contexto conturbado da saúde pública mundial, a Conferência Internacional sobre Cuidados Primários de Saúde, reunida em Alma-Ata, em 1978 foi um dos estertores para se realizar uma reforma nos sistemas de saúde de diversos países, inclusive o Brasil. Alma-Ata expressou a necessidade de ação urgente para promoção da saúde da população mundial, tendo reforçado a nova definição da OMS - saúde como estado de completo bem-estar físico, mental e social, e não simplesmente a inexistência de moléstias - sendo um direito imprescindível. Almejava-se o progresso econômico para a mais completa realização da meta de "Saúde para Todos no Ano 2000" a fim de se atenuar a desigualdade (MINISTÉRIO DA SAÚDE, 2002).

A Conferência enfatizou a importância dos cuidados primários de saúde, constituindo a chave para que esta meta fosse atingida. Estes representam o primeiro nível de contato com o sistema de saúde, pelo qual os cuidados são levados o mais proximamente possível aos lugares onde as pessoas vivem e trabalham, constituindo o primeiro elemento de um continuo processo de assistência (MINISTÉRIO DA SAÚDE, 2002). Indubitavelmente, é importante salientar que a luta pela instituição desse sistema universal ficou conhecido por "Movimento Sanitarista".

No Brasil, esse movimento foi denominado Reforma Sanitária ganhando força dentro das Universidades com propostas de reivindicações que foram catalisadas na $8^{\underline{a}}$ Conferência Nacional de Saúde em 1986. As resoluções dessa Conferência deram os parâmetros que mais tarde viriam a ser inscritos na Constituição de 1988 (MINISTÉRIO DA SAÚDE, 2000). A Reforma Sanitária Brasileira foi proposta num momento de intensas mudanças, cobiçando-se que pudesse servir à democracia e à consolidação da cidadania no país (ESCOLA NACIONAL DE SAÚDE PÚBLICA SÉRGIO AROUCA, 2009; FALEIROS VP, et al., 2006). Nesse contexto, em outubro de 1988, com a promulgação da nova Constituição Federal, completou-se o processo de retorno 
do país ao regime democrático e abriu-se o caminho para considerar a saúde um direito de cidadania, originando assim ao processo de criação de um sistema público, universal e descentralizado de saúde: o Sistema Único de Saúde - SUS (PAIVA CHA, TEIXEIRA LA, 2014).

Como visto, outrora a saúde era entendida como o "Estado de não doença", o que fazia com que toda lógica girasse em torno das doenças e agravos à saúde, remediando apenas os efeitos e com menos ênfase nas causas. Essa lógica deu lugar a uma nova convicção centrada na prevenção das doenças e na promoção de saúde, mudando o conceito do termo para "estado de bem-estar físico, social e mental" (MINISTÉRIO DA SAÚDE, 2000).

Assim, o SUS delineou seus princípios e diretrizes tendo como referência os atributos essenciais que definem e fundamenta a Atenção Primária a Saúde (APS). Começando pela acessibilidade, que envolve a imediação de localização do serviço, horários de funcionamento, possibilidade de admissão de consultas sem agendamento prévio, questiona-se até que ponto esses aspectos são percebidos como pertinentes pela população (AQUINO R, et al., 2014).

O termo "primeiro contato" subentende-se pela acessibilidade e utilização dos serviços de saúde pelos usuários frente a um novo contratempo ou a cada novo episódio de um mesmo transtorno (PAIM JS, 2009). Assim, pode ser entendido como porta de entrada nos serviços, isto é, quando a população e a equipe identificam aquela prestação de serviço como o primeiro meio a ser buscado quando necessário (CONSELHO NACIONAL DE SECRETÁRIOS DE SAÚDE, 2011).

Já a longitudinalidade expressa a existência de uma fonte regular de atenção e de um profissional responsável pelo cuidado com o paciente, além de registro de informações e seu acesso e uso ao longo do tempo, independente do agravo à saúde, bem como a vivência em relação a isso (PAIM JS, 2009).

O termo "continuidade do cuidado", no que concerne à bibliografia internacional, tem sido utilizado como semelhante ao atributo longitudinalidade, apesar de possuírem especificidades em suas acepções - mesmo que interrupções na continuidade da atenção aconteçam, não necessariamente deverá deixar de existir a relação pessoal de longa duração entre profissionais de saúde e usuários (AQUINO R, et al., 2014).

A integralidade deve fazer com que o paciente receba todos os tipos de cuidados essenciais nos três níveis de atenção à saúde (primário, secundário e terciário), não devendo ser restringido a serviços de alto nível de complexidade.

A coordenação abrange a implementação de vínculos entre a equipe da APS e as outras instituições de diferentes níveis de complexidade, com base na cooperação e na comunicação, de modo que a atenção básica se transforme na entidade de ligação entre os cidadãos e os outros elementos do sistema de saúde (AQUINO R, et al., 2014).

Além dos atributos essenciais, têm-se também os derivados. A orientação familiar consiste no sistema de apoio que o indivíduo está inserido e seu potencial de cuidado na saúde de cada membro.

A orientação comunitária refere-se ao entendimento de que cada população específica tem seu contexto social que pode influir no processo de saúde. A competência cultural trata-se da capacidade das equipes em reconhecer as abundantes peculiaridades e necessidades específicas de cada população, que podem variar conforme a etnia, raça, religião, entre outros (CONSELHO NACIONAL DE SECRETÁRIOS DE SAÚDE, 2011; MINISTÉRIO DA SAÚDE, 2010).

Com o objetivo de avaliar a qualidade da APS, foi criado o Instrumento de Avaliação da Atenção Primária (PCATool - PrimaryCare Assessment Tool) (MINISTÉRIO DA SAÚDE, 2010).

Esse instrumento foi desenvolvido por uma equipe de trabalho no "The Johns Hopkins Populations Care Policy Center", órgão que percebeu a APS como um dos pilares para uma boa saúde pública (HARZHEIM E, et al., 2006).

O questionário em questão apresenta versões em sua origem atribuídas a crianças, adultos que são maiores de 18 anos, idosos e também, profissionais de saúde. Mensura assim, a presença e o desdobramento 
dos quatro atributos essenciais (acesso de primeiro contato, longitudinalidade, integralidade, coordenação da atenção) e dos três atributos derivados (orientação familiar, orientação comunitária e competência cultural) (MINISTÉRIO DA SAÚDE, 2010).

Para encaixá-lo na realidade brasileira, versões do questionário foram adaptadas de forma que pudessem ser usadas como entevista. Houve então um processo de adequação e legitimação de conteúdo, além de estudo de confiabilidade (HARZHEIM E, et al., 2006).

A adaptação foi realizada em dois momentos: primeiro foi traduzido do inglês para o português e depois deste para o inglês novamente. No final, comparou-se a versão original em inglês com a obtida pela tradução reversa, reparando as falhas.

O questionário original foi desenvolvido para ser auto-aplicado e foi adaptado para ser realizado por intermédio de entrevistadores, sendo também preciso adaptá-lo às realidades socioculturais e à idade da população abrangida. No quesito confiabilidade, foram realizados vários testes, em intervalo médio de 24 dias, comparando-se o resultado dos atributos obtidos em dois momentos distintos, através de testes estatísticos (HARZHEIM E, et al., 2006).Com o aumento da população, consequentemente ascende também à problemática da saúde, caracterizando o aumento de agravos à mesma que requerem cuidados continuados.

Com isso, é importante reconhecer a nova realidade da população brasileira e se atentar para questões sobre a qualidade oferecida pelo SUS aos usuários, principalmente em relação aos atributos que regem esse sistema. Dessa forma, justifica-se a avaliação desses atributos sob o olhar dos cidadãos, a fim de se corrigir falhas no sistema, além de implementar políticas de saúde local, medidas preventivas e intervenções baseadas na equidade.

\section{MÉTODOS}

Após aprovação do projeto pelo Comitê de Ética e Pesquisa da Universidade de Franca, sob o no 3.804.818, foi realizado um estudo analítico, transversal caracterizado pela coleta de dados por meio da aplicação do questionário PCATool - Brasil versão adulto, por três alunas do curso de medicina, em usuários de cinco Unidades Básicas de Saúde da cidade de Franca, estado de São Paulo.

As Unidades possuem parceria com a Universidade de Franca e o instrumento de avaliação foi aplicado na sala de espera das mesmas no período de agosto a novembro de 2018.

Foram selecionados aleatoriamente 20 pacientes em cada UBS, na faixa etária dos 18 aos 59 anos, de ambos os sexos, totalizando 100 indivíduos. Foi utilizado como critério de inclusão estar entre a faixa etária abrangida pelo questionário, além de aceitar participar da pesquisa por meio da assinatura do Termo de Consentimento Livre e Esclarecido.

Como critério de exclusão estavam os adultos que não atendiam às características citadas anteriormente e aqueles que não podiam responder por si mesmos ou que apresentavam dificuldade clara de entendimento em relação aos instrumentos utilizados na coleta.

As entrevistas foram feitas utilizando-se um questionário com perguntas sociodemográficas (idade e sexo) e outras para avaliação da Atenção Primária à Saúde (PCATool - Brasil) versão Adulto, validado no Brasil pelo Ministério da Saúde.

Este instrumento se propõe a avaliar a estrutura e o processo de atenção nos serviços de Atenção Básica tomando por base os atributos essenciais e derivados da APS, sendo eles: atributos essenciais [grau de afiliação com o serviço de saúde; acesso de primeiro contato (dimensões utilização e acessibilidade); longitudinalidade; coordenação da atenção (dimensões: integração de cuidados e sistemas de informações); integralidade (dimensões: serviços disponíveis e serviços prestados)] e atributos derivados (orientação familiar e orientação comunitária). $\mathrm{Na}$ análise estatistica, as variáveis quantitativas idade e escores foram descritas em relação aos parâmetros média-aritmética e seus respectivos desvios-padrões. 
Tendo o teste de normalidade de D'Agostino e Pearson sugerido populações de origem normalizadas a comparação dos escores entre os grupos correspondentes a cada Unidade Básica estudada, foi realizada análise de variância paramétrica de Fisher.

Para analisar a variável qualitativa sexo em cada grupo foi utilizada a estatística de Qui-quadrado $\left(\mathrm{X}^{2}\right)$. $\mathrm{O}$ nível de significância pré estabelecido foi de $5 \%(\alpha=0,05)$ e os cálculos foram realizados no software GraphPad Prism 5.0. Os gráficos foram elaborados no aplicativo Excel do Office 2010.

\section{RESULTADOS}

Em todas as Unidades Básicas avaliadas o número de mulheres entrevistadas foi maior do que o de homens, totalizando $77 \%$ de mulheres entrevistadas e 33\% de homens (Gráfico 1).

Gráfico 1 - Relação entre os sexos.

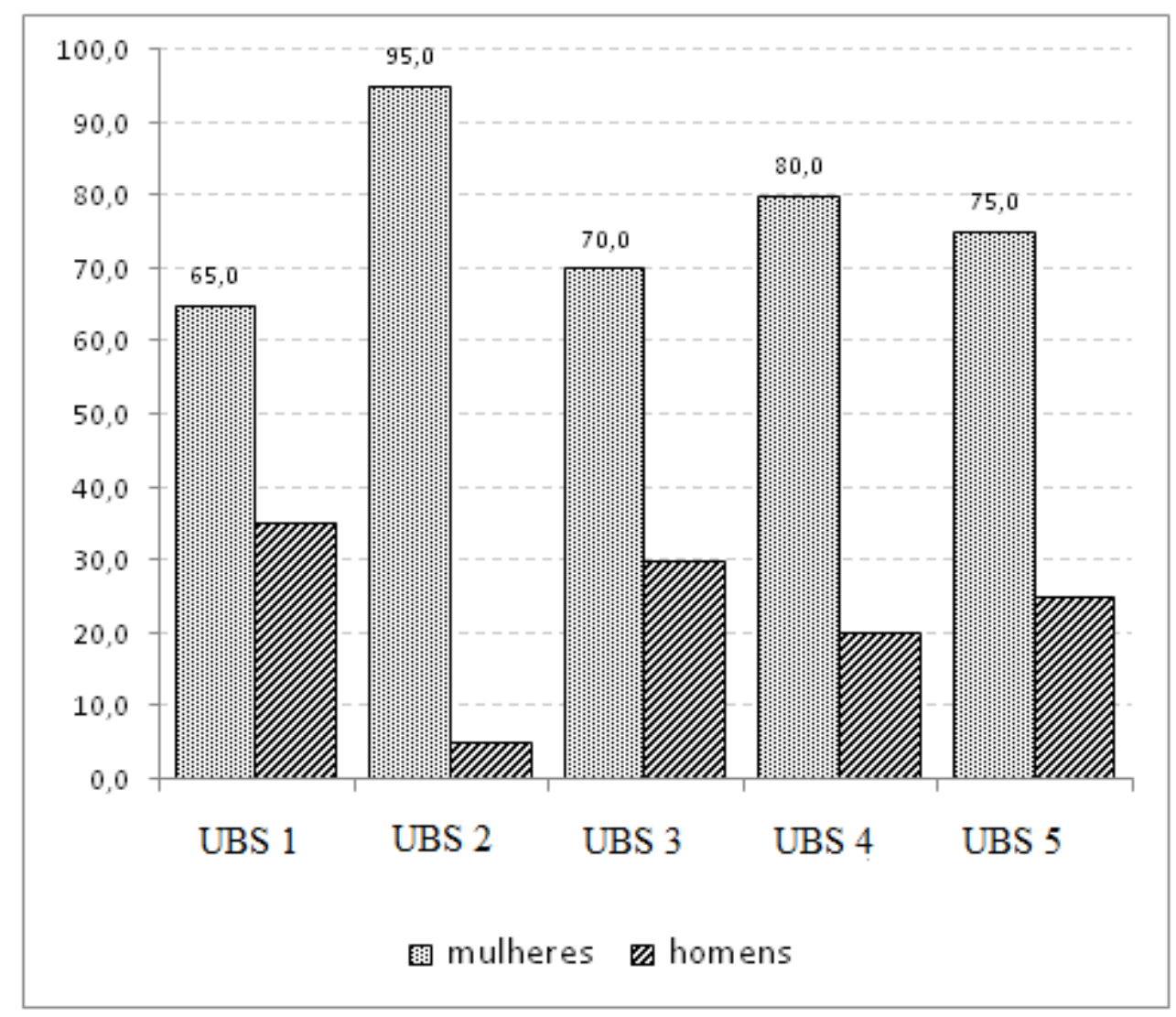

Fonte: Morais ICO, et al, 2020.

A média das idades foi de 39 anos na UBS 1; 34 anos na UBS 2; 44 anos na UBS 3; 35 anos na UBS 4 e 39 anos na UBS 5, constituindo uma média total de 38 anos entre as cinco unidades básicas

Em relação aos atributos, não foram encontradas diferenças significativas entre os escores. O pior escore encontrado foi a Integralidade - dimensão serviços prestados (escore médio - 2,11) e o único atributo orientado para a APS foi o acesso de primeiro contato, com a dimensão utilização (escore médio - 7,88) (Tabela 1).

O escore essencial é obtido pela soma do escore médio dos elementos que pertencem aos atributos essenciais enquanto o grau de afiliação, dividido pelo número de elementos deste atributo (Gráfico 2). Por fim, o escore geral é alcançado pela soma do escore médio dos elementos constituintes dos atributos essenciais, somado aos que pertencem aos atributos derivados mais grau de afiliação, dividido pelo número total de itens presentes no questionário (MINISTÉRIO DA SAÚDE, 2010) (Gráfico 3). 
Tabela 1 - Médias dos escores e p valores.

\begin{tabular}{|c|c|c|c|}
\hline Atributos e dimensões & Média & Desvio-padrão & P valor \\
\hline Afiliação & 4,86 & 2,76 & 0,11 \\
\hline Utilização & 7,88 & 2,31 & 0,882 \\
\hline Acessibilidade & 2,87 & 1,2 & 0,27 \\
\hline Longitudinalidade & 4,68 & 1,85 & 0,41 \\
\hline Integração & 3,03 & 2,55 & 0,07 \\
\hline Sistema de informações & 5,7 & 2,23 & 0,46 \\
\hline Serviços disponíveis & 5,44 & 1,3 & 0,21 \\
\hline Serviços prestados & 2,11 & 2,12 & 0,77 \\
\hline Orientação farmiliar & 2,86 & 2,55 & 0,58 \\
\hline Orientação comunitária & 2,27 & 1,79 & 0,39 \\
\hline Escore essencial & 4,7 & 1,15 & 0,73 \\
\hline Escore geral & 4,3 & 1,13 & 0,91 \\
\hline
\end{tabular}

Fonte: Morais ICO, et al, 2020.

Gráfico 2 - Escore Essencial.

\section{Escore essencial}

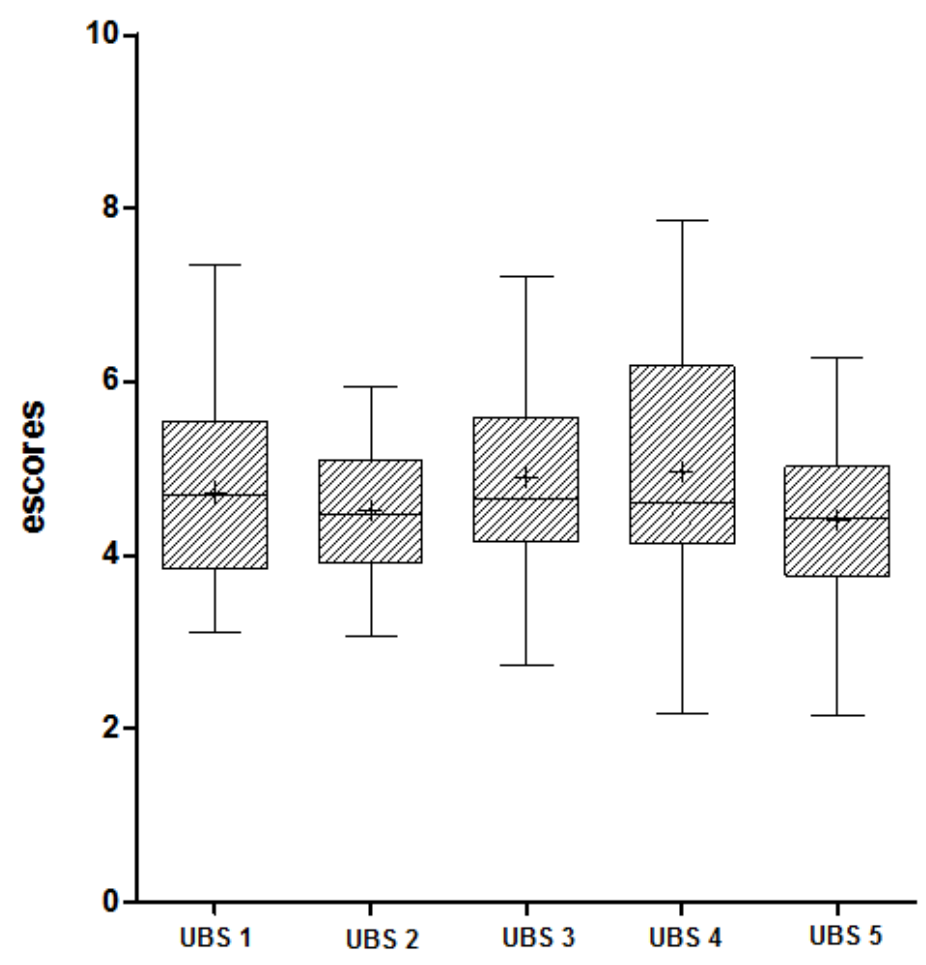

Fonte: Morais ICO, et al, 2020. 
Gráfico 3 - Escore geral.

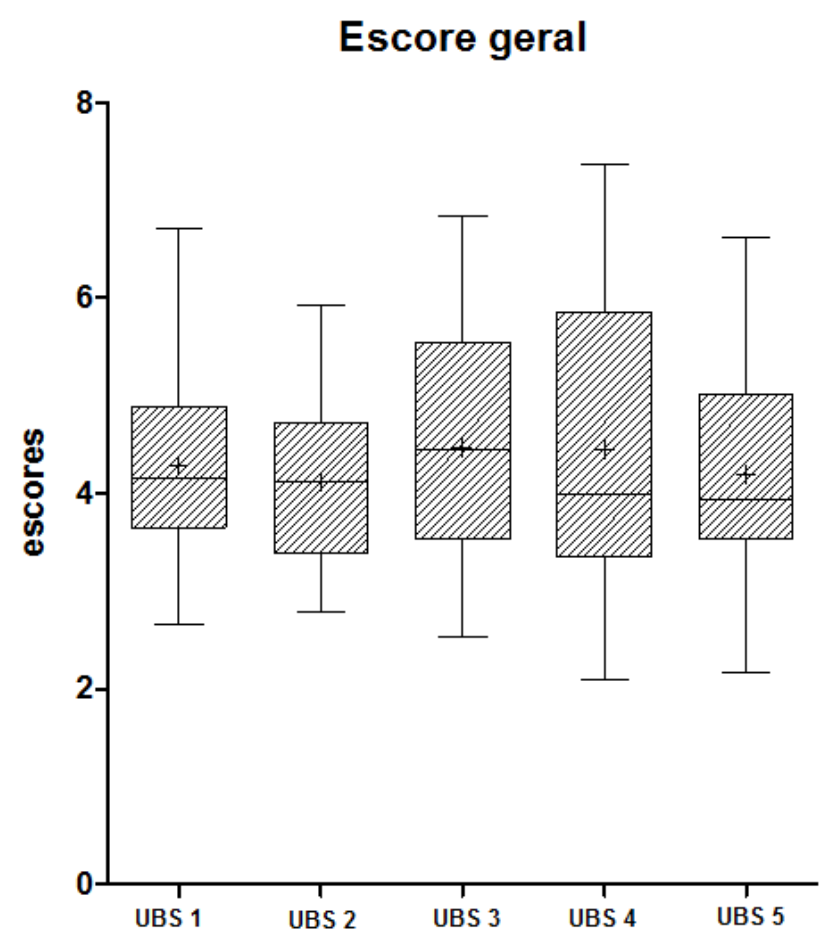

Fonte: Morais ICO, et al, 2020.

\section{DISCUSSÃO}

A saúde pública é um dos principais deveres do Estado para com a população, sendo forte motivo de incerteza para aqueles quea utilizam. Este como detentor do poder tem a obrigação de elaboração e efetuação de estratégias que limitem os riscos e agravos à saúde, garantindo a acessibilidade e equidade aos indivíduos ao SUS em seus diferentes níveis de atenção (CARVALHO G, 2013). Apesar de o Estado empenhar-se nesse contexto, mantém-se perceptível o alto índice de desigualdade no tocante aos cuidados à saúde, tendo como etiologias diferenças de raça, situação econômica e a desigualdade de gênero.

Indubitavelmente, é perceptível que os serviços de saúde têm oferecido os mesmos tipos de serviços e cuidados aos homens e mulheres, sendo que são indivíduos que necessitam de atenção diferenciada, visto os modelos discrepantes de adoecimento. No presente estudo é possível constatar que o sexo feminino tem maior participação na procura por atendimento. Tais relações socioculturais estabelecidas desenham a idéia de que o homem tende a considerer o acesso à saúde como sinônimo de fragilidade e de que os serviços de saúde costumam ser identificados como locais de mulheres, crianças e idosos (LEVORATO CD, et al., 2014). Isso é interpretado como uma das possibilidades relacionadas à ínfima participação do gênero masculino na procura por atendimento à saúde, já que não consideram a doença inerente à sua própria condição biológica.

A maior participação feminina do estudo reflete também as estatísticas brasileiras de que as mulheres são maioria na população e que estas adoecem mais (realidade ligada mais ao cenário de discriminação do que as condições biológicas) e que além de buscar os cuidados próprios nestes serviços, muitas vezes os frequentam como cuidadoras dos demais familiares (MINISTÉRIO DA SAÚDE, 2008).

Além disso, a procura por atendimento somente após um agravo da morbidade tem como consequência atraso na Atenção e maiores custos. Esta resistência aumenta também o sofrimento físico e emocional, visto que tratamentos crônicos têm menor adesão. (MINISTÉRIO DA SAÚDE, 2008). Isso fica evidente após constatação por pesquisas demonstrando que a mortalidade proporcional por grupo etário a partir dos 15 anos de idade é maior em homens do que em mulheres (MINISTÉRIO DA SAÚDE, 2014). 
A APS é um modelo de sistematização que objetiva ser a porta de entrada ao sistema de saúde, integrando os diferentes níveis de atenção e englobando serviços como promoção de saúde, prevenção de agravos, diagnóstico precoce, tratamento e reabilitação. Esta tem competência para resolução de cerca de $85 \%$ dos problemas em saúde da população (CONSELHO NACIONAL DE SECRETÁRIOS DE SAÚDE, 2011). A APS é responsável pela resolução dos problemas de saúde mais frequentes, considerando cada usuário em sua individualidade e particularidades, dentro do contexto sociocultural abrangido (SHIMAZAKI ME, 2009).

O reconhecimento da importância da APS é fundamentado em evidências de seu impacto na saúde e desenvolvimento de países que a utilizam. Dentre os atributos essenciais, o primeiro contato consiste na utilização do serviço de saúde a cada evento de saúde - o primeiro local a ser frequentado quando surge alguma necessidade (OLIVEIRA MAC, PEREIRA IC, 2013).

Esse princípio também abrange a acessibilidade, que inclui o acesso geográfico (localização do local de atendimento, meios de transporte para se chegar ao local) e o acesso sócio-organizacional (horários de atendimento, demora para conseguir consultas, filas, entre outros) - componentes que podem dificultar 0 acesso ao atendimento (OLIVEIRA MAC, PEREIRA IC, 2013).

Apesar de o escore utilização ter obtido pontuação acima da média, fora perceptível no estudo que falta conhecimento da população a respeito dos diferentes níveis de atenção e quais locais buscar ao surgir algum problema, pois foi notório que muitos usuários ainda procuram serviços emergências por questões que seriam resolutivas na APS.

Ademais, o componente acessibilidade obteve um escore bem inferior ao esperado, principalmente por questões como o não funcionamento durante o período noturno e aos finais de semana e tempo de demora para conseguir consultas. Pode-se também relacionar essa questão com a discrepância entre os gêneros que foi observada, já que os homens ainda são a maioria da população no mercado de trabalho e os serviços de saúde não possuem horários acessíveis para os trabalhadores, tal como o funcionamento aos finais de semana e período noturno.

Habitualmente, as equipes de APS vêm se acomodando em função de metas predeterminadas, por meio de protocolos e diretrizes preconizados pelo Ministério da Saúde, fato este que reprime bastante o reconhecimento do caráter transversal da saúde do trabalhador (SILVA TL, et al., 2013).

Ao abordar a Longitudinalidade compreende-se o acompanhamento no decorrer do desenvolvimento de cada indivíduo e suas mudanças ao longo dos anos. Pauta-se na relação interpessoal que se constrói entre pacientes e equipe de saúde e, para isso, é necessária confiança mútua entre usuário e profissionais de saúde, fato este que, devido aos resultados obtidos, ainda não é evidenciado.

Contudo, deve ser almejado visto que propicia menos doenças preveníveis e atenção mais oportuna e adequada, pois, ao criar vínculo com os profissionais, o paciente costuma seguir mais suas recomendações (DOTTO JM, et al., 2016). A grande rotatividade das equipes prejudica o estabelecimento desse vínculo almejado (CONSELHO NACIONAL DE SECRETÁRIOS DE SAÚDE, 2011).

Os usuários postularam durante as entrevistas que geralmente a rotatividade médica era elevada, o que não possibilita a criação de um cuidado longitudinal, diferentemente dos demais profissionais da saúde que geralmente são uma equipe fixa. $O$ quesito integralidade engloba a capacidade de uma equipe multidisciplinar de identificação e resolução dos problemas de um indivíduo, seja resolvendo-os na APS ou através de encaminhamento (SHIMAZAKI ME, 2009).

Novamente foi perceptível que o maior empecilho foi o escasso conhecimento pelos usuários acerca de quais os serviços disponíveis na APS, pois muitos responderam que serviços que são oferecidos pelos demais níveis de atenção, tais como sutura de ferimentos, são oferecidos na Unidade Básica de Saúde. É evidente a falta de comunicação entre os trabalhadores de saúde e usuários, não só nas unidades referentes a pesquisa em questão, mas em âmbito nacional. Não é incomum encontrar campo infértil para o desenvolvimento de habilidades para comunicar-se, uma vez que, a própria lógica dos serviços oferecidos não favorece o estabelecimento de relações saudáveis (OLIVEIRA A, et al., 2008). 
Porém, em relação ao atributo serviços prestados, escore que obteve menor pontuação, foram unânimes os feedbacks sobre nunca terem sidos abordados em relação a questões de prevenção, tais como uso de cinto de segurança, como guardar medicamentos em segurança, entre outros. O atributo Coordenação do Cuidado é indispensável para o sucesso dos demais. Sem ele, a longitudinalidade perde muito do seu potencial, a integralidade é inviável e o primeiro contato acaba por tornar-se meramente administrativo.

Este atributo é avaliado pelo componente "sistema de informação", o qual também não atingiu o mínimo necessário para considerar que estava orientado para APS. Pode-se inferir que ainda há necessidade de melhora da qualidade de informação, seja por melhores mecanismos de comunicação ou implantação de prontuários eletrônicos que conectem os diferentes níveis de atenção, por exemplo (SHIMAZAKI ME, 2009).

Dentre os atributos derivados, a abordagem familiar postula que a equipe de saúde reconheça a importância do contexto familiar nos cuidados da saúde dos indivíduos. Quando se conhece esse contexto os resultados obtidos são mais satisfatórios já que se estabelece um melhor vínculo com o paciente, favorecendo a corroboração quando forem necessários investigar e intervir em alguma situação (SHIMAZAKI ME, 2009). Foi notório também que esse escore permaneceu aquém do esperado.

A orientação comunitária é a capacidade da equipe de reconhecimento das características e peculiaridades da população que frequenta determinada unidade básica, identificando quais problemas são mais comuns, resolvendo-os, incentivando a participação social no planejamento das ações e buscando informações a respeito da satisfação dos usuários (SHIMAZAKI ME, 2009). A despeito disso, obteve-se um escore baixo, pois muitos entrevistados não possuíam conhecimento acerca de visitas domiciliares, apesar de serem feitas, e também por questões tais como nunca terem sido interrogados sobre a satisfação com 0 funcionamento da unidade, além de nunca terem sido convidados para participar do Conselho Gestor.

\section{CONCLUSÃO}

Ainda existem inúmeras barreiras para o aperfeiçoamento das ações na APS analisando a realidade e necessidades de saúde da população brasileira. Apesar dos resultados obtidos em relação à qualidade da APS em cinco Unidades Básicas não terem sido favoráveis, percebe-se que muito dos problemas abordados seriam resolvidos facilitando o acesso dos usuários aos serviços, capacitando às equipes multidisciplinares sobre o que abordar na APS, além de educação da população a respeito do funcionamento do Sistema Único de Saúde para que este realmente funcione de forma organizada e com destreza. Indubitavelmente, conscientizando a população a respeito dos diferentes níveis de atenção e também facilitando o acesso a esses serviços de forma que inclua a todos, os cidadãos teriam uma melhor percepção da APS como porta de entrada e, dessa forma, possibilitaria um dos maiores objetivos desse tipo de atenção: promover saúde e prevenir doenças.

\section{REFERÊNCIAS}

1. AQUINO R, et al. A Estratégia Saúde da Família e o reordenamento do sistema de serviços de saúde. In: Paim, J.S; Almeida Filho, N. (Org). Saúde Coletiva: teoria e prática. Rio de Janeiro: Med Book, 2014. P. 353-371.

2. CARVALHO G. A saúde pública no Brasil. Estud av [Internet]. 2013.

3. CONSELHO NACIONAL DE SECRETÁRIOS DE SAÚDE. Atenção Primária e Promoção da Saúde: Coleção para Entender a Gestão SUS. Brasília: Tatiana Rosa; 2011.

4. DOTTO JM, et al. Avaliação da qualidade dos serviços de atenção primária à saúde acessados por idosos em dois distritos de Porto Alegre, RS, Brasil. Revista da Faculdade de Odontologia. 2016; 21(1): 23-30.

5. ESCOLA NACIONAL DE SAÚDE PÚBLICA SÉRGIO AROUCA. Assistência Farmacêutica: em Foco no Estado do Rio de Janeiro. Rio de Janeiro: Fiocruz; 2009.

6. FALEIROS VP, SILVA JFS, VASCONCELLOS LCS, SILVEIRA RMG. A Construção do SUS: Histórias da Reforma Sanitária e do Processo Participativo. Brasília: MS; 2006.

7. HARZHEIM E, STARFIELD B, Rajmil L, ÁLVARES-DARDET C, STEIN AT. Consistência interna e confiabilidade da versão em português do Instrumento de Avaliação da Atenção Primária (PCATool-Brasil) para serviços de saúde infantil. Cad Saúde Pública. 2006; 22(8): 1649-1659. 
8. LEVORATO CD, MELLO LM, SILVA AS, NUNES AP. Fatores associados à procura por serviços de saúde numa perspectiva relacional de gênero. Ciência e Saúde Coletiva. 2014; 19(04).

9. MINISTÉRIO DA SAÚDE. Declaração de Alma Ata sobre Cuidados Primários. BVMS.

10. MINISTÉRIO DA SAÚDE. Manual do Instrumento de Avaliação da Atenção Primária à Saúde [monografia na internet]. Brasília: Ministério da Saúde; 2010.

11. MINISTÉRIO DA SAÚDE. Política Nacional de Atenção Integral à Saúde do Homem- Princípios e Diretrizes [Internet]. Brasília: Ministério da Saúde;

12. MINISTÉRIO DA SAÚDE. Política Nacional de Atenção Integral à Saúde da Mulher - Princípios e Diretrizes [Internet].

13. MINISTÉRIO DA SAÚDE. Saúde Brasil 2014 Uma análise da situação de saúde e das causas externas. Brasília: Ministério da Saúde; 2015

14. MINISTÉRIO DA SAÚDE. SUS Princípios e Conquistas. BVMS [periódicos na internet], 2000.

15. OLIVEIRA A, et al. A comunicação no contexto do acolhimento em uma unidade de saúde da família de São Carlos, São Paulo. Botucatu, 2008.

16. OLIVEIRA MAC,PEREIRA IC.Atributos essenciais da Atenção Primária e a Estratégia Saúde da Família Rev Bras Enferm. 2013; 66(esp): 158-64.

17. PAIM JS. O Que é o SUS. Rio de Janeiro: Fiocruz, 2009.

18. PAIVA CHA, TEIXEIRA LA. Reforma Sanitária e a Criação do Sistema Único de Saúde: notas sobre contextos e autores. Hist Cienc Saúde Manguinhos 2014 Mar; 21(1):15-35.

19. SHIMAZAKI ME. A Atenção Primária à Saúde. Escola de Saúde Pública do Estado de Minas Gerais. Belo Horizonte: 2009.

20. SILVA TL, et al. Saúde do trabalhador na Atenção Primária: percepções e práticas de equipes de Saúde da Família. Botucatu; 2013. 